\title{
DESENVOLVIMENTO DE UM MEDIDOR INTELIGENTE DE BAIXO CUSTO PARA APLICAÇÃO EM SMART GRID COMO CASO DE APRENDIZAGEM BASEADA EM PROBLEMA
}

Alexandre Magnus Fernandes Guimarães - alexandremagnus@ect.ufrn.br

Universidade Federal do Rio Grande do Norte, Escola de Ciências e Tecnologia

Avenida Senador Salgado Filho, 3000

59078-970 - Natal - Rio Grande do Norte

Gabriel de Melo Souza-gabrieldemelosouza@gmail.com,

Rhendson Alexandre Ferreira - rhendson.alexandre@hotmail.com

Universidade Federal do Rio Grande do Norte, Departamento de Engenharia Elétrica

Avenida Senador Salgado Filho, 3000

59078-970 - Natal - Rio Grande do Norte

Resumo: Com o aumento da demanda energética e da geração distribuída, as redes elétricas passaram a ser pensadas como redes elétricas inteligentes. Nesse contexto, concessionárias e consumidores residenciais necessitam de formas mais eficientes e menos onerosas de mensurar e avaliar a energia elétrica. Diante disto, o presente artigo objetiva relatar o processo de pesquisa envolvido no desenvolvimento de um medidor inteligente baseado em um CI de aquisição de dados energéticos e um módulo Wi-Fi (ESP32), utilizando-se da metodologia de aprendizagem baseada em problema, que visa a detecção e resolução do problema concernente à questão energética atual através do produto construído. Como resultado, o presente estudo viabilizou a consolidação de um protótipo funcional atendendo às expectativas de integração às redes elétricas a um baixo custo. Por fim, o presente relato de pesquisa concluiu que o desenvolvimento de projetos acadêmicos impacta profundamente na inovação tecnológica, atingindo os mais diversos atores sociais.

Palavras-chave: Aprendizagem baseada em problema. Sistemas embarcados. Redes elétricas inteligentes. Medidores inteligentes.

\section{INTRODUÇÃO}

O atual crescimento da demanda de energia elétrica leva as concessionárias e os governos a dispenderem maior esforço nas tentativas de acompanhar as tendências. Uma projeção divulgada no Plano Decenal de Expansão de Energia (EPE, 2019), prevê um crescimento de $3,9 \%$ ao ano no consumo residencial de energia. As concessionárias têm de lidar também com o crescimento da geração distribuída, impulsionada pela queda nos preços de painéis solares (PORTAL SOLAR, 2020), e no maior aproveitamento dos recursos energéticos renováveis, como a energia eólica. Assim, as concessionárias têm de repensar como as redes elétricas são organizadas, já que a estrutura habitual não comportará as mudanças de forma adequada.

Segundo Momoh (2012), as redes elétricas inteligentes diferem das redes elétricas atuais ao possuírem melhores esquemas de comunicação e novas técnicas de medição para aumentar a resiliência e conseguir conter perturbações internas e externas. Logo, a nova rede é capaz de melhor suportar eventos de transferência de energia não agendados entre regiões e prover um fornecimento com maior confiabilidade e qualidade. 
Os medidores inteligentes são uma ferramenta fundamental no desenvolvimento e implementação das redes elétricas inteligentes, também chamadas de smart grids. Ainda de acordo com Momoh (2012), os medidores inteligentes ou smart meters dão informações aos consumidores sobre o quanto consomem e o preço que é cobrado por kWh, o que resulta em contas mais precisas e uma detecção mais rápida das quedas de energia. Além disso, o consumidor pode ter acesso ao seu perfil de consumo (quando e com quais aparelhos a energia elétrica é consumida) e participar de programas para o consumo reduzido e mais inteligente de energia elétrica. Outros aspectos da utilização de medidores inteligentes também incluem o desligamento à distância de eletrodomésticos, por exemplo.

Diante do exposto, o presente trabalho tem como objetivo principal relatar a pesquisa concernente ao desenvolvimento de um medidor inteligente de baixo custo que possa atuar para atender as seguintes necessidades:

1. Dos consumidores residenciais/industriais, para que obtenham dados de utilização e que possam traçar seu perfil de consumo;

2. Da concessionária, fornecendo dados da quantidade e qualidade (presença de harmônicas, queda de fase, curtos-circuitos, etc.) da energia.

O desenvolvimento dos smart meters contribui no campo da inovação e impulsiona o crescimento das redes elétricas inteligentes. Ainda dentro do contexto dos medidores inteligentes, o desenvolvimento dos mesmos dentro deste projeto contribui com o conhecimento acadêmico para todos os estudantes envolvidos, tornando-os profissionais mais completos e aptos a atuar com sucesso neste ramo da engenharia elétrica.

Como forma de orientar este trabalho, foi utilizado o método de aprendizagem baseada em problema. Segundo Lambros (2004) Aprendizagem Baseada em Problema (ABP) consiste em um método de ensino que utiliza problemas como ponto de partida na aquisição de novos conhecimentos. Segundo Duch et al. (2001), a metodologia de ABP dá aos estudantes novas habilidades quanto à busca de soluções para um problema e os ajuda a construir o conhecimento de forma diferente da maneira usual, permitindo-os melhor retê-lo e aplicá-lo de forma mais eficiente.

Como ponto de partida para o desenvolvimento do medidor inteligente, foram considerados os problemas gerados pela maior demanda por energia elétrica. Através da utilização de ABP, foram obtidos pelos desenvolvedores, como benefícios, a aprendizagem e aperfeiçoamento dos conceitos de sistemas embarcados, programação embarcada e de aplicação, banco de dados, eletrônica e elétrica (alta potência).

Este relato de pesquisa é estruturado em seis diferentes partes, visando facilitar a compreensão do leitor sobre os diversos aspectos que compõem o desenvolvimento do produto proposto. Primeiramente, na introdução, aborda-se o contexto no qual as redes elétricas inteligentes estão inseridas e os desafios que são impostos aos agentes envolvidos na situação, além de delinear os objetivos gerais do presente relato de pesquisa e a maneira como a mesma foi desenvolvida. Na segunda parte, são explicitadas as especificações dos medidores inteligentes e os componentes utilizados em sua construção. Em seguida, na terceira parte, são explicados o design do circuito e do hardware. Logo após, na quarta parte, é realizada a análise das quantidades mensuradas pelo medidor. Na quinta parte, expõe-se a programação do sistema embarcado e do software de exibição de dados ao usuário. Finalmente, na sexta e última parte do presente trabalho serão abordadas as conclusões sobre a pesquisa e o desenvolvimento do proposto medidor e como este colabora na solução dos problemas levantados. 


\section{ESPECIFICAÇÕES DO MEDIDOR}

A primeira etapa do desenvolvimento do medidor consiste em determinar as quantidades a serem mensuradas. $\mathrm{O}$ medidor inteligente fornecerá informações sobre o consumo, a tensão da rede e a qualidade de energia; isso se traduz em valores RMS de tensão e corrente, potências, fatores de potência e presença e intensidade de frequências harmônicas (ou seja, múltiplas da frequência de $60 \mathrm{~Hz}$ da rede brasileira). A importância da medição dessas frequências reside na determinação da qualidade da energia e nas interferências geradas na rede elétrica por equipamentos como motores. O medidor também deve suportar um sistema trifásico para atender diferentes tipos de consumidores.

São considerados, como requisitos, que o medidor seja alimentado pela tensão da rede e possua conectividade $\mathrm{Wi}-\mathrm{Fi}$, para que possa transmitir facilmente os dados registrados. É necessário que, além de ser um medidor de fácil instalação e leitura, tenha baixo custo, para que seja acessível a uma vasta gama de consumidores.

\subsection{Escolha dos componentes}

Para o processamento de dados de tensão e corrente, foi escolhido o circuito integrado ADE7880, do fabricante Analog Devices ( $\mathrm{mm}$ ), ele é certificado por diferentes normas (ANALOG DEVICES, 2014), assegurando boa confiabilidade e tornando-o ideal para aplicações comerciais.

A escolha do circuito integrado foi motivada pelo uso prévio de seu antecessor, o CI ADE7758, que já havia sido explorado e testado com sucesso (GUIMARÃES et al., 2015). O CI ADE7880 amplia as funcionalidades do CI ADE7758, fornecendo informações sobre todas as quantidades medidas em relação à frequência fundamental e total (por exemplo, potência ativa da fase A, total e somente da fundamental), além de diversas medições sobre a presença de frequências harmônicas na rede. Essas medições podem ser utilizadas para obter informações sobre a qualidade da energia e definir como equipamentos elétricos provocam perturbações (frequências harmônicas) na rede. O CI ADE7880 também apresenta um menor erro de medição e é considerado como a solução atual da Analog Devices para medição de energia. O CI fornece todas as quantidades desejadas para sistemas monofásicos e trifásicos (delta e estrela), sendo elas: valores eficazes de tensão e corrente, potências (ativa, reativa e aparente), detecção de picos e quedas de tensão, além de outras medições que podem ser exploradas futuramente.

O controle do sistema é feito com um ESP32, que possui Wi-Fi integrado e é um dos mais conhecidos controladores no mercado de Internet das Coisas (IoT). Seu núcleo opera a uma frequência de $240 \mathrm{MHz}$ e permite fácil integração com um sistema operacional em tempo real, permitindo uma aquisição e transmissão síncrona dos dados em intervalos préestabelecidos de 100 milissegundos. Devido à facilidade de montagem e baixo custo, foi utilizada neste projeto uma placa de desenvolvimento do ESP32, o módulo ESP32 DOITDevKit V1.

Para alimentação, utiliza-se o módulo HLK-PM03 da Hi-Link, que é um conversor AC/DC e aceita uma tensão alternada de 110-220V em entrada, além de fornecer os 3,3V necessários para o CI ADE7880 e para o módulo ESP32 na saída. Quanto à medição da corrente, foi utilizado um sensor não invasivo baseado em um transformador de corrente (CT), o SCT-013 100A.

Tomando como base o valor do dólar a $\mathrm{R} \$ 5,20$ e os orçamentos feitos para a aquisição de 100 peças, estima-se que o valor total da parte eletrônica do projeto é de R $\$ 143,86$, discriminado na tabela 1 . O valor é relativamente baixo quando comparado a medidores comerciais, que custam em média $\mathrm{R} \$ 2.000,00$. Os valores considerados são para pequenas 
"Os desafios para formar hoje o engenheiro do amanhã"

quantidades de componentes, ou seja, podem ser reduzidos caso sejam fabricados medidores em larga escala.

Tabela 1 - Preços e quantidades dos componentes eletrônicos.

\begin{tabular}{|c|c|c|}
\hline Componente & Quantidade & Preço unitário (R\$) \\
\hline ADE7880 & 1 & 54,70 \\
\hline ESP32 DOIT-DevKit V1 & 1 & 19,97 \\
\hline HLK-PM03 & 1 & 16,36 \\
\hline SCT-013 100A & 3 & 23,67 \\
\hline PCB & 2 & 4,16 \\
\hline Outros (resistores, capacitores, cristais) & - & 25 \\
\hline \multicolumn{2}{|c|}{ Total } & 143,86 \\
\hline
\end{tabular}

Fonte: Elaborado pelo autor (2020) com base nos sites aliexpress.com e mouser.com.br.

Após a definição de todos os componentes foi traçado o esquema de blocos do sistema, que mostra os componentes do medidor em linhas gerais. O esquema é mostrado na Figura 1. A partir do exposto será traçado o esquemático do circuito na seção seguinte.

O bloco "Sensoriamento e Condicionamento dos Sinais de Tensão e Corrente" é composto pelos sensores de corrente acompanhados de dois circuitos sugeridos pelo datasheet e pelas notas de aplicação. O primeiro circuito serve para transformar a corrente obtida pelos sensores em uma tensão a ser lida pelo circuito integrado; enquanto o segundo serve para converter a tensão de $220 \mathrm{~V}$ em um nível que possa ser lido pelo circuito integrado, que possui uma tensão máxima de entrada de $0,25 \mathrm{~V}$.

Figura 1 - Esquema de blocos do sistema.

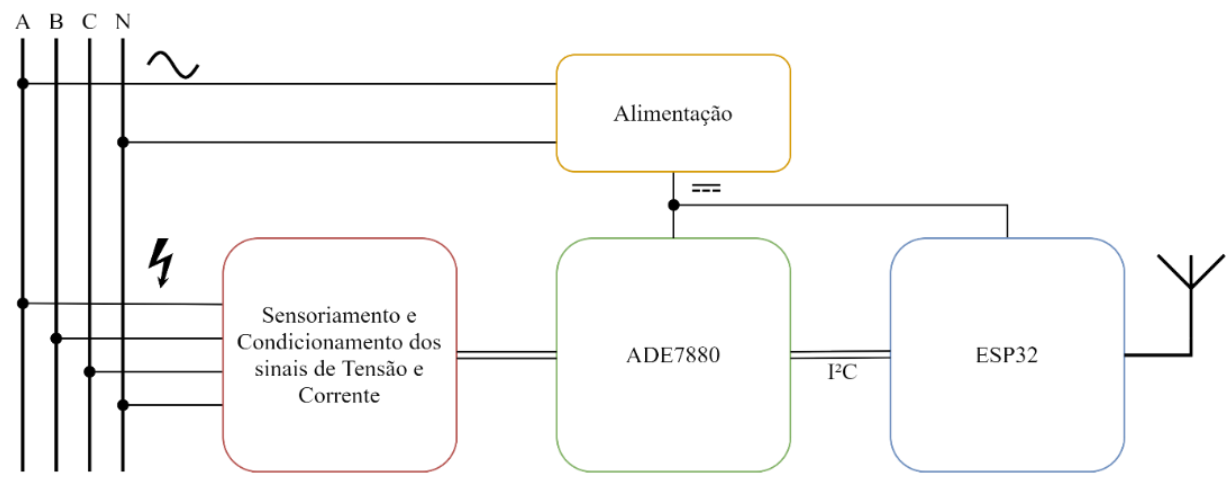

Fonte: Elaborado pelo autor (2020).

\section{DESENVOLVENDO O HARDWARE}

A partir do desenvolvimento do hardware, é possível se aproximar da concretização de um produto funcional. Para tanto, o circuito do CI ADE7880 é montado como descrito no datasheet; isso inclui todos os componentes necessários, como capacitores e osciladores. Foram utilizados componentes SMD, que são o padrão atual para as novas placas utilizadas na indústria (MONKMAN, 2002), visando reduzir o tamanho do produto final e seguir o layout sugerido no datasheet (ANALOG DEVICES, 2014a).

Por fim, é realizado o design do bloco de condicionamento e sensoriamento. A tensão máxima em cada uma das entradas analógicas do CI ADE7880 é de 500mV; enquanto a tensão da rede a ser medida, é de 220Vrms (ou 311,12Vpico). Para adaptá-la, utiliza-se um 
divisor de tensão com uma razão de 1:1000, como sugerido em uma das notas de aplicação (ANALOG DEVICES, 2014b). A Figura 2 mostra a entrada analógica do circuito integrado.

Figura 2 - Entrada analógica do circuito integrado.

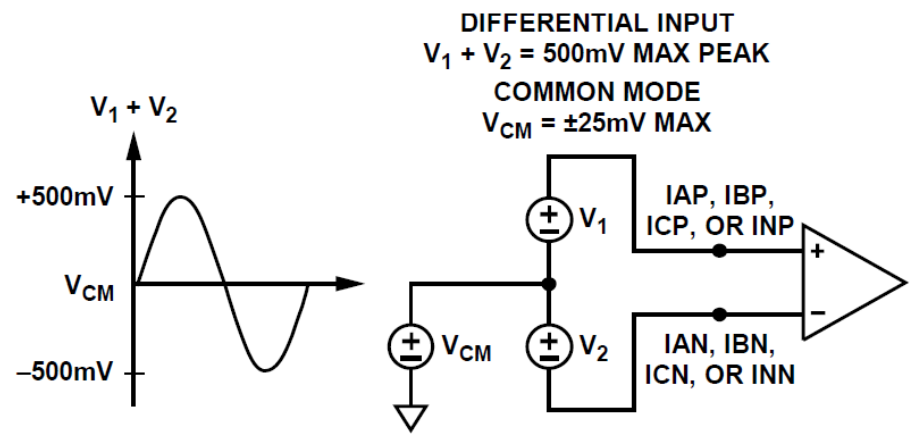

Fonte: ANALOG DEVICES (2014a).

A Figura 3A mostra o divisor de tensão desenvolvido, que acompanha um filtro passabaixa RC com uma frequência de corte de $5 \mathrm{kHz}$. Essa frequência é suficiente para monitorar as principais harmônicas e manter longe todas as réplicas criadas pela amostragem de 1.024 MHz. O indutor L1 é uma ferrita que serve como filtro de entrada.

A corrente de entrada vem de um transformador de corrente (SCT-013 100A) que é convertida em tensão através dos resistores de carga, mostrados na figura 3B (R30 e R32). O circuito possui o mesmo filtro passa-baixa mencionado anteriormente. A conversão em tensão também se baseia nos limites mostrados na Figura 2.

Figura 3 - A: Divisor de tensão. B: Conversão de corrente em tensão

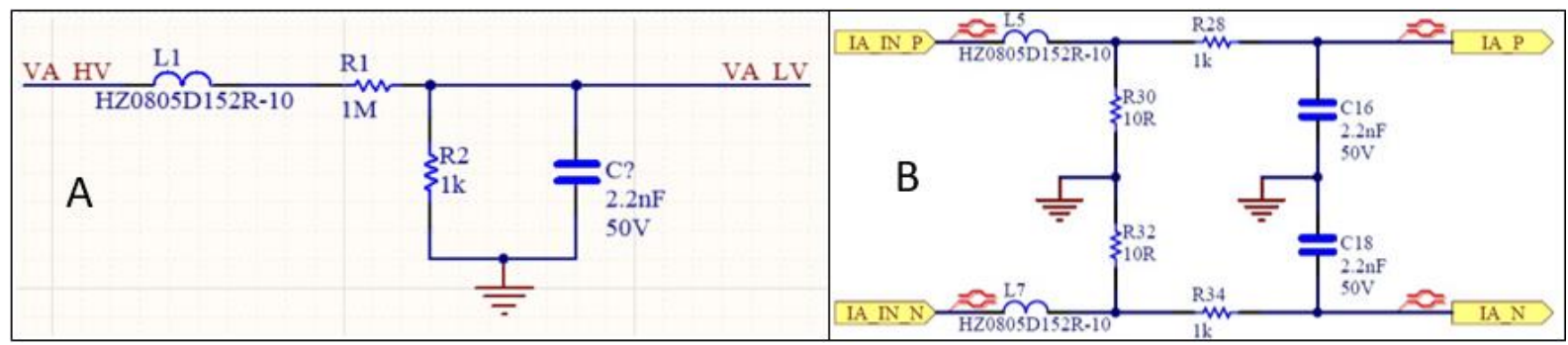

Fonte: Elaborado pelo autor (2020).

As duas placas, já com os componentes anteriormente mencionados conectados, são mostradas na Figura 4. Para a maior segurança do usuário, toda a parte de alta tensão está em uma placa de circuito impresso separada, incluindo o módulo Hi-Link.

A placa de circuito impresso foi desenvolvida utilizando o software Altium Designer®. A placa principal, contendo o CI ADE7880 e o módulo ESP32, tem dimensões de 6,75 x 7,00 $\mathrm{cm}$; a placa secundária, contendo o circuito em alta tensão, possui $5,00 \times 6,50 \mathrm{~cm}$. Ambas foram fabricadas em uma placa de FR-4 de duas camadas.

O módulo ESP32 possui diversas interfaces de comunicação com o CI ADE7880: uma para recuperar os dados e para configurar o circuito integrado $\left(\mathrm{I}^{2} \mathrm{C}\right)$; outra para visualizar as formas de onda de tensão e corrente (HSDC); outra para configurar o modo de consumo de energia (ou power mode); e finalmente uma interface que gera interrupções caso ocorra um evento, como uma queda de energia. Devido ao seu tamanho e à necessidade de economia de espaço na placa, o circuito integrado se encontra embaixo do módulo ESP32. 


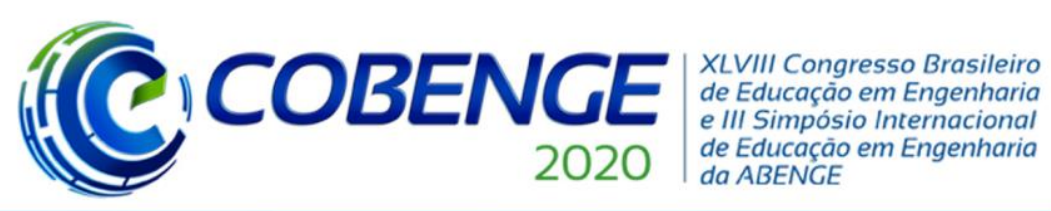

"Os desafios para formar hoje o engenheiro do amanhã"

Figura 4 - Hardware finalizado do produto.

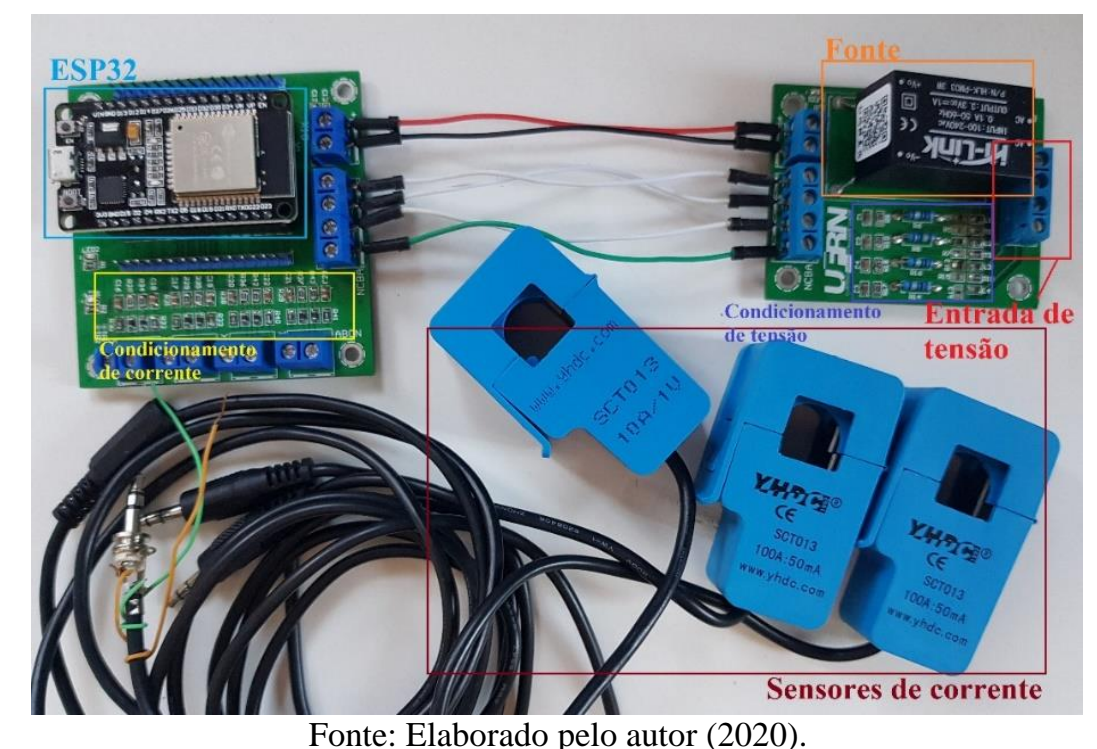

\section{MEDINDO QUANTIDADES}

O CI ADE7880 é o componente do hardware responsável pelas medições. A partir dele, é possível obter os valores eficazes (ou RMS) de tensão e corrente, potências (ativa e aparente), fator de potência e frequências harmônicas. O valor eficaz pode ser interpretado como a tensão que seria fornecida caso a fonte fosse contínua ao invés de alternada (RICO, 2006). O cálculo do valor RMS de um sinal discreto, segundo a Equação 1, leva em conta a frequência fundamental da rede e suas harmônicas, gerando um problema, pois é necessário medir qual é a energia que é consumida efetivamente (através da frequência fundamental) e qual é a influência das harmônicas na rede. Para resolver esse problema, o CI ADE7880 utiliza um filtro passa-baixa digital integrado no circuito.

$$
F_{R M S}=\sqrt{\frac{1}{T} \int_{0}^{T} f^{2}(t) \mathrm{d} t}=\sqrt{\frac{1}{T} \int_{0}^{T} F \sin ^{2}(\omega t+\alpha) \mathrm{d} t}
$$

Como mostrado na equação 1 , o sinal $f$, que pode ser de tensão ou de corrente, possui uma forma senoidal com uma amplitude $\mathrm{F}$ e uma fase $\alpha$. $\mathrm{O}$ cálculo da potência aparente é dado pela multiplicação entre os valores eficazes de tensão e de corrente. A potência ativa é a potência aparente multiplicada pela diferença de fase entre a tensão e a corrente, como mostrado na Equação 2.

$$
P_{\text {ativa }}=P_{\text {aparente }} \cdot \cos \left(\alpha_{V}-\alpha_{I}\right)=V_{R M S} \cdot I_{R M S} \cdot \cos \left(\alpha_{V}-\alpha_{I}\right)
$$

Com relação às harmônicas, é possível obter diversos dados (potência ativa, reativa e aparente, fator de potência, valores eficazes e distorção harmônica) de qualquer um dos múltiplos dos $60 \mathrm{~Hz}$ na rede a partir das leituras do CI ADE7880. Isso garante que o usuário tenha uma vasta gama de informações, atendendo a uma grande diversidade de aplicações possíveis. As informações obtidas podem ser lidas e armazenadas por um software dedicado. 


\section{SOFTWARE EMBARCADO E LEITURA DOS RESULTADOS}

A principal função do software embarcado é garantir o funcionamento contínuo, ou seja, a coleta das amostras em intervalos de tempo pré-fixados de 100 milissegundos. As informações são enviadas ao cliente logo após serem coletadas. Além da função de coleta de dados, o medidor deve ser expansível de acordo com as demandas do cliente, funcionando como um relé ou recebendo interrupções vindas do CI ADE7880, para que ele possa agir como disjuntor em caso de curto-circuito ou desligamento à distância de equipamentos.

O intervalo de 100 milissegundos foi escolhido para que seja possível monitorar todas as variações na rede. Além disso, espera-se também que mesmo seja suficiente para adquirir as amostras e enviá-las por MQTT (um intervalo menor causaria congestionamentos na transmissão). Caso ocorra algum surto que não seja detectado devido ao intervalo, o CI ADE7880 enviará uma interrupção ao microcontrolador avisando-o do ocorrido.

O Messsage Queuing Telemetry Transport, ou MQTT, é um protocolo de transmissão de mensagens via internet ideal para a comunicação entre máquinas (M2M) e internet das coisas (IoT). Seu sistema de publish e subscribe é amplamente utilizado devido à sua escalabilidade. O MQTT também tem diversas vantagens sobre o HTTP, como por exemplo: melhor segurança, melhor tempo de resposta, melhor taxa de transferência, e, mais importante para Internet das Coisas, menor consumo de bateria e de largura de banda (ERIDANI et al., 2019). O protocolo MQTT é integrado no módulo Wi-Fi (ESP32).

Para cumprir as funcionalidades descritas anteriormente, utiliza-se um sistema operacional em tempo real (RTOS, Real-Time Operating System), o freeRTOS. Um RTOS funciona como um sistema operacional de computadores, mas para sistemas embarcados. Nele, temos duas tarefas principais:

- A tarefa de aquisição, responsável por ler os dados do ADE7880 e transmiti-los à tarefa de comunicação;

- A tarefa de comunicação, responsável por transmitir os dados via Wi-Fi, utilizando o protocolo MQTT.

A Figura 5 mostra um fluxograma do software embarcado. Nele estão contidas as principais etapas e verificações pelas quais o produto passa. Primeiramente, o controlador, juntamente com suas entradas e saídas, é iniciado; em seguida os protocolos de rede, e, por fim, o CI de aquisição de dados. Uma vez adquiridas as medições necessárias, o sistema as envia ao cliente e espera o intervalo pré-fixado para realizar outra medida.

Para programar o software, foi utilizado o datasheet do ESP32 como referência (ESPRESSIF SYSTEMS, 2020), juntamente com as bibliotecas fornecidas pelo fabricante (ESP-IDF). O software foi programado em linguagem C, utilizando a IDE Visual Studio Code com o auxílio do CMake.

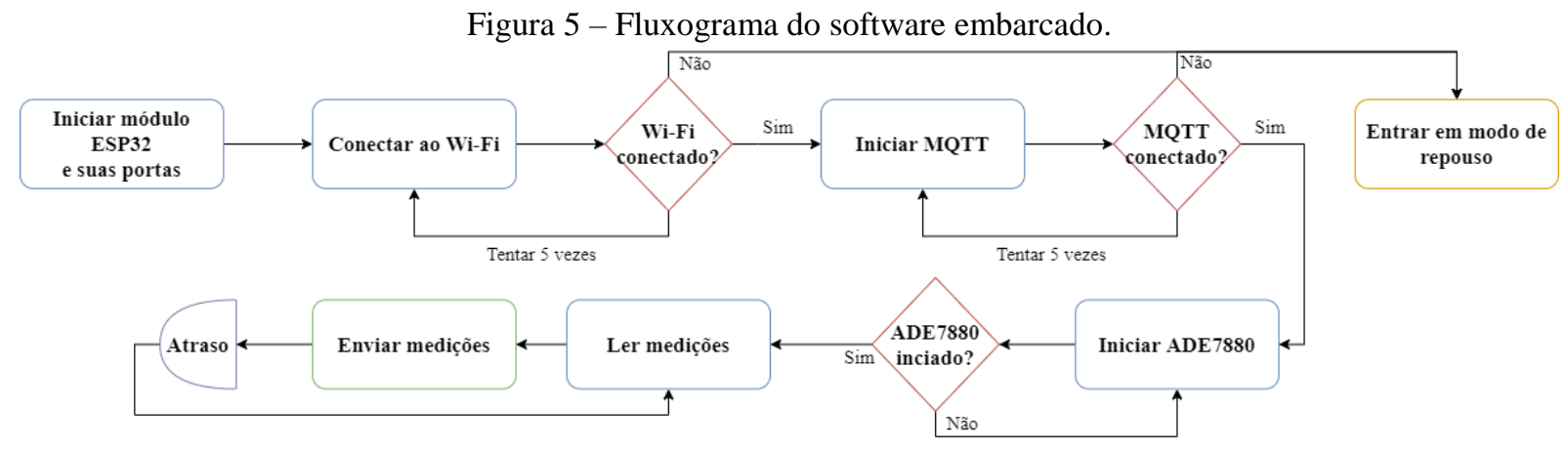

Fonte: Elaborado pelo autor (2020). 
A recuperação e a visualização dos dados do medidor são feitas através do Node-RED, que é uma ferramenta de programação visual criada pela IBM Emerging Technology, voltada para IoT (LEKIC e GARDASEVIC, 2018). O Node-RED pode inscrever-se em tópicos (categorias de mensagem) e receber os dados publicados pelo ESP32. Utiliza-se o broker (servidor) público do Eclipse, com usuário e senha a fim de proteger os dados do cliente.

Os dados recebidos pelo Node-RED ficam armazenados em um banco de dados SQLite, e assim podem ser analisados posteriormente para a contestação de contas de eletricidade ou mesmo para monitoramento do consumo.

O Node-RED também permite a criação de uma interface gráfica para monitorar os dados, além de poder ser facilmente executado a partir de um terminal. Visando o baixo custo, pode-se utilizar um módulo Raspberry Pi dedicado a essa tarefa. A Figura 6 mostra a interface gráfica desenvolvida, exibida em um navegador web (Google Chrome). Apesar de estar em sua versão inicial, apenas exibindo valores eficazes de tensão e corrente, a interface permite escolher entre a visualização de diversos medidores.

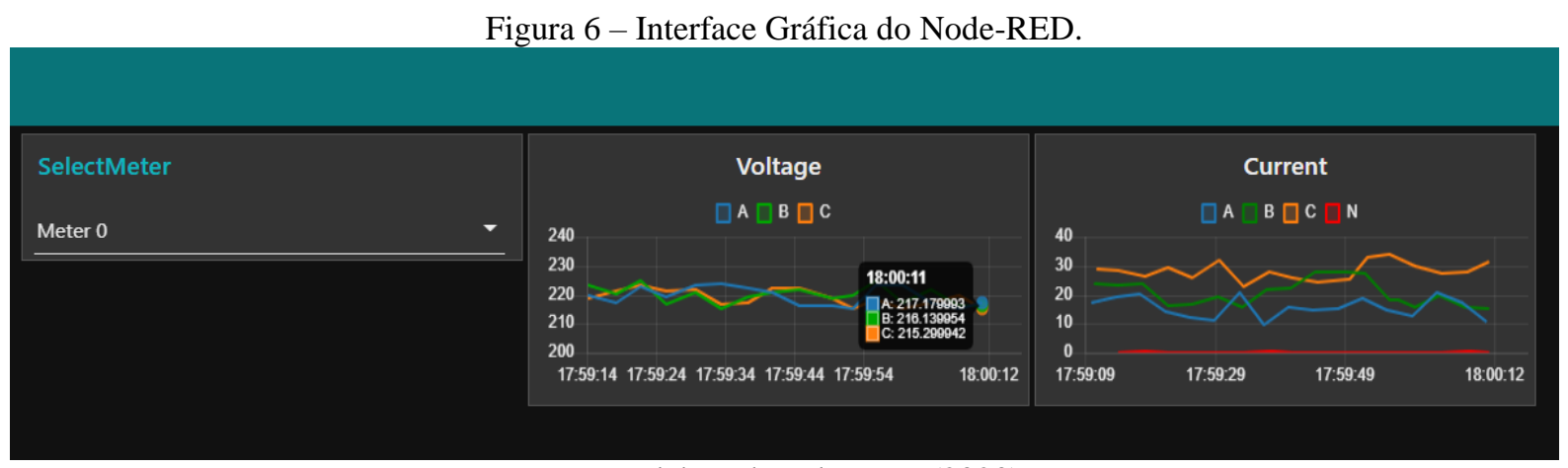

Fonte: Elaborado pelo autor (2020).

\section{RESULTADOS E CONCLUSÃO}

O presente estudo teve como objetivo relatar a pesquisa realizada no desenvolvimento de um medidor inteligente e de baixo custo, voltado para a aplicação em smart grid, visando solucionar os problemas provenientes da crescente demanda energética no Brasil.

O estudo descreve todas as etapas do desenvolvimento do medidor conforme ocorrem, eventualmente consolidando a construção do mesmo. A primeira etapa consiste em transformar as especificações no diagrama de blocos do sistema, permitindo a escolha dos componentes. Em seguida, foi concebido o hardware (placa de circuito impresso), que contém os componentes anteriormente mencionados e que permite a programação da aplicação. Posteriormente, analisa-se mais a fundo as quantidades a serem medidas, para que sejam corretamente programadas e recuperadas do circuito integrado. Finalmente, são descritos o software embarcado e o protótipo da aplicação desenvolvida.

Assim, o estudo atingiu seu objetivo ao desenvolver o medidor inteligente, sendo possível programar o hardware desenvolvido e recuperar as medidas mencionadas anteriormente. Estas são recuperadas pela aplicação Node-RED e corretamente exibidas ao usuário. O medidor, dessa forma, está apto a atender às necessidades dos consumidores residenciais e das concessionárias, como proposto. Além disso, o desenvolvimento do produto cumpre o objetivo de funcionar como método de aprendizado aos estudantes envolvidos, que fixaram conceitos relevantes em sistemas embarcados, eletrônica, programação, smart grids, smart meters, entre outros, utilizando-se do método de ABP.

$\mathrm{O}$ presente relato de pesquisa conclui que o desenvolvimento de projetos no âmbito acadêmico, utilizando-se de metodologias pré-estabelecidas, como a ABP, são cruciais para a 
fixação de conhecimentos dos alunos envolvidos, preparando-os para desenvolver mais projetos que estejam atentos às necessidades e problemas enfrentados, para que possam levar a inovação tecnológica aos diversos atores socias.

A principal limitação do presente estudo reside nos desafios impostos pelo isolamento social causado pela pandemia do novo coronavírus. Isolamento este que impossibilitou a validação completa do medidor, conforme descrita em GUIMARÃES et al. (2015). Este tipo de validação e comparação com medidores comerciais requer a utilização de diversas cargas resistivas, indutivas e capacitivas, de diversos níveis de tensão e configurações de rede diferentes. Os instrumentos de validação, como osciloscópios e fontes de tensão, somente estão disponíveis nos laboratórios da universidade, cujo acesso era impossibilitado. Dessa forma, foi realizada somente a validação parcial do produto, nas residências onde o acesso era possível.

Para ampliar as discussões e enriquecer o campo de pesquisas sobre os medidores inteligentes recomenda-se o desenvolvimento de novos estudos que validem as medidas obtidas e as comparem com medidores residenciais. Além disso, seria engrandecedora para a área a expansão das funcionalidades dos medidores inteligentes, para que estes pudessem funcionar também como disjuntores, fazendo uso de relés ligados aos pinos do ESP32 que não estão sendo utilizados; ou a utilização de algoritmos de Machine Learning para processar a grande quantidade de dados disponíveis e prever surtos na rede.

\section{REFERÊNCIAS}

ANALOG DEVICES. ADE7758 Datasheet. 2004. Disponível em:

https://www.analog.com/media/cn/technical-documentation/data-sheets/ADE7758.pdf.

Acesso em: 31 jul 2020.

ANALOG DEVICES. ADE7880 Datasheet. 2014a. Disponível em:

https://www.analog.com/media/en/technical-documentation/data-sheets/ADE7880.pdf.

Acesso em: 16 jun 2020.

ANALOG DEVICES. Application Note: Impact of Adding a Neutral Attenuation Network in a 3P4W Wye System. 2014b. Disponível em https://www.analog.com/media/en/technicaldocumentation/application-notes/AN-1334.pdf. Acesso em: 16 jun 2020.

DUCH, Barbara J.; GROH, Susan E.; ALLEN, Deborah E. The Power of Problem-Based Learning: A Practical "How-To" for Teaching Undergraduate Courses in any Discipline. Sterling, Viriginia: Stylus. 2001.

EMPRESA DE PESQUISA ENERGÉTICA (EPE). Plano Decenal de Expansão de Energia. Disponível em: https://www.epe.gov.br/sites-pt/publicacoes-dadosabertos/publicacoes/PublicacoesArquivos/publicacao-422/PDE\%202029.pdf. Acesso em: 10 jun. 2020.

ERIDANI, Dania; MARTONO, Kurniawan T.; HANIFAH, Amaniyya A. MQTT Performance as a Message Protocol in na IoT based Chili Crops Greenhouse Prototyping. In: International Conference on Information Technology, Information Systems and Electrical Engineering, 4., 2019, Indonésia. Proceedings. Indonésia, 2019. 
"Os desafios para formar hoje o engenheiro do amanhã"

ESPRESSIF SYSTEMS. ESP32 Datasheet. 2020. Disponível em:

https://www.espressif.com/sites/default/files/documentation/esp32_datasheet_en.pdf. Acesso em: 19 jun. 2020.

GUIMARÃES, A. M. F.; FREITAS, T. T.; GRINER, H.; DE ALMEIDA, T. H. S. Smart Energy Monitoring System with ADE7758 IC. In: International Youth Conference on Energy (IYCE), 5., 2015, Pisa, Itália. Proceedings. Pisa, Itália, 2015.

LAMBROS, Ann. Problem-Based Learning in Middle and High School Classrooms: A Teacher's Guide to Implementation. Thousand Oaks: Corwin Press, Inc. 2004.

LEKIC, Milica; GARDASEVIC, Gordana. IoT sensor integration to Node-RED platform. In: International Symposium INFOTEH-JAHORINA, 17., 2018, Bósnia e Herzegovina. Proceedings. Bósnia e Herzegovina, 2018.

MOMOH, James. Smart Grid: Fundamentals of Design and Analysis. $1^{\text {a }}$ edição, Hoboken: John Wiley \& Sons, Inc, 2012.

MONKMAN, Gareth. Problems of Scale. Assembly Automation, v.22, n.1, p. 8-9, 2002.

PORTAL SOLAR. Preço dos painéis solares cai 90\% em nove anos. Portal Solar, 2020.

Disponível em https://www.portalsolar.com.br/blog-solar/energia-solar/preco-dos-paineissolares-cai-90-em-nove-anos.html. Acesso em: 18 jun 2020.

RICO, Guillermo. The Technology Interface, v.6, n. 1, 2006.

YHDC. SCT-013 Datasheet. Disponível em https://www.mcielectronics.cl/website_MCI/static/documents/Datasheet_SCT013.pdf. Acesso em: 16 jun 2020.

\title{
DEVELOPMENT OF A LOW-COST SMART METER FOR SMART GRID APPLICATIONS AS A PROBLEM-BASED LEARNING CASE
}

\begin{abstract}
With the rising energy demand and microgeneration, electrical grids are now conceived as smart grids. In this context, electric utility and residential consumers need more efficient and less costly ways to measure and evaluate electricity. In view of this, this paper aims to report the research process involved in developing a smart meter based on an energy metering IC and a Wi-Fi module (ESP32), using the problem-based learning methodology, which aims at the detection and resolution of the problem concerning the current energy matter through the build product. As a result, the present study made it possible to consolidate a functional prototype meeting the electrical grid integration expectations at a low cost. Finally, the present research report concluded that the development of academic projects has a profound impact on technological innovation, reaching the most diverse social actors.
\end{abstract}

Keywords: Problem-based learning. Embedded systems. Smart grid. Smart meters. 